\title{
Bacterial microbiota composition of fresh unpasteurized cow's milk and home-made and commercially available fermented milk products.
}

\author{
Pieter de Waal ${ }^{1}$, Shane Murray ${ }^{2}$, Katie Viljoen ${ }^{3}$, Jeanne Korsman² ${ }^{2}$ and Michael Levin ${ }^{1}$ \\ ${ }^{1}$ University of Cape Town \\ ${ }^{2}$ Centre of Proteomic and Genomic Research \\ ${ }^{3}$ University of Cape Town Faculty of Health Sciences
}

February 12, 2021

\begin{abstract}
Background: Rural communities who consume unpasteurized and traditional fermented milk products on a regular basis, have a low prevalence of allergic diseases. Lactic acid producing bacteria present within these products, is postulated to have an allergy protective role against atopy. Objective: To characterize and compare the bacterial microbiota of fresh unpasteurised cow's milk and to explore the effect of milk fermentation (commercially and traditionally fermented) on the bovine milk microbiota. Methods: Raw, unpasteurized cow's milk was collected from urban and rural farms. Another sample, collected from a rural farm, was left to ferment naturally. Three different brands of commercially fermented milk samples were also analysed. The V3 and V4 regions of the 16S rRNA gene were amplified to assess microbiota composition. Results: Urban and rural fresh milk had the highest microbiota alpha diversity, and commercially bought fermented milk products, the least. Commercially fermented milk was consistently dominated by lactic acid producing bacteria, belonging to the phylum Firmicutes, while homemade fermented milk comprised of approximately 50\% Firmicutes and 50\% Proteobacteria. The relative abundance of several organisms differed between fermented and unfermented milk. Lactococcus lactis dominated all milk products, however its relative abundance was lower in fresh milk compared with fermented milk. Lactobacillus paracasei and Streptococcus infantis were abundant in traditionally fermented milk, but absent in commercially fermented products. Potential pathogens were demonstrated in fresh and home fermented milk. Conclusion: Commercially fermented milk can be promoted as a safe and possible allergy protective complementary feed from 1 year of age.
\end{abstract}

Conflict of Interest The authors declare no potential conflict of interests related to the manuscript content.

\section{Funding Source}

Research Grant - Allergy Society of South Africa, South Africa.

\section{Key words}

Microbiota, biodiversity, fermentation, cow's milk, amasi, allergy prevention, rural.

To the Editor,

The anti-allergic properties of raw and fermented cow's milk are being explored globally. ${ }^{1,2}$

Traditionally fermented milk (isiXhosa - 'amasi') and unfermented (unpasteurized) cow's milk (isiXhosa 'ubisi '), are consumed regularly by rural South African communities. To produce amasi, unpasteurized milk is left for three to five days to naturally ferment at room temperature. The South African Food Sensitisation and Food Allergy (SAFFA) study, demonstrated that children living in an urban environment had 
significantly higher rates of allergic diseases compared to their rural counterparts, and the consumption of fermented milk was associated with lower rates of allergic rhinitis, atopic dermatitis and self-reported asthma. ${ }^{3}$ Lactic acid producing bacteria produce important by-products and end-products during milk fermentation which may contribute to their anti-inflammatory and anti-allergic properties. ${ }^{4}$ In this study we characterize and compare the bacterial microbiota in raw cow's milk (collected from urban and rural farms), and homemade and commercially fermented milk products by high throughput $16 \mathrm{~S}$ rRNA gene amplicon sequencing. This study received ethical approval from the University of Cape Town (animal ethics: 018_033).

Rural fresh cow's milk samples were collected from three farms in rural South Africa. Urban samples were collected from a farm in Cape Town. Before milking commenced, the cows and the udders were declared in a "heathy state" by each farmer. The udders were not cleaned or washed prior to milking, as these procedures were not included in the normal milking routine on the farms. Samples were labelled as Rural fresh cow's milk 2, Rural fresh cow's milk 3, Urban fresh cow's milk 1, Urban fresh cow's milk 2 and Urban fresh cow's milk 3 and transported frozen to the laboratory. A further sample of unpasteurized milk obtained from a separate farm in the same rural area labelled as Home fermented milk (amasi), was sealed, and left for five days at ambient temperatures to allow natural fermentation. Three different brands of commercially fermented amasi were obtained and labelled as Comm-othando, Comm-amyoli andComm-maas. All samples were analyzed by the Centre for Proteomic and Genomic Research (CPRG), Cape Town, South Africa.

DNA was extracted from the milk samples using the ZymoBIOMICS@ DNA Miniprep Kit (Zymo Research, USA). The V3-V4 variable region of the $16 \mathrm{~S}$ rRNA gene was amplified from $2.5 \mathrm{ng}$ to $25 \mathrm{ng}$ of purified DNA

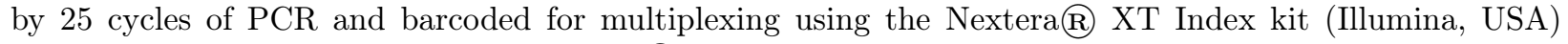
and KAPA HiFi DNA Polymerase (Rocheß, USA). The nine milk product samples, a positive control (ZymoBIOMICS\&. Microbial Community DNA standard) and a negative control (DNA suspension buffer) were included in library preparation. The size of the libraries was verified using an Agilent $® 2100$ Bioanalyzer (Agilent, USA). Library concentration was evaluated using the KAPA Illumina Library Quantification Kit (Roche). The libraries were sequenced on an Illumina MiSeq sequencer at the CPRG using a MiSeq Reagent Kit v2 (Illumina $囚)$ ) to produce paired-end 250 base pair reads.

Illumina MiSeq read quality assessment and taxonomic profiling were performed on a high-performance compute cluster using a custom Nextflow pipeline [https://github.com/h3abionet/TADA], implementing FastQC ${ }^{5}$ and MultiQC ${ }^{6}$ for quality control, dada $2^{7}$ for ASV prediction, and the RefSeq-RDP 16 S database (v3 May 2018) for taxonomic annotation. ${ }^{8}$ All downstream analyses were performed in R, with custom functions [https://gist.github.com/kviljoen/97d36c689c5c9b9c39939c7a100720b9].Taxa (merged at the lowest available taxonomic level, tax_glom.kv function) were deemed significantly different (in terms of abundance and/or absence/presence) between fermented versus unfermented samples if they exhibited a fold change (beta coefficient) of [?] 1.5 and had an adjusted p-value of [?] 0.05 and if at least one of the two groups compared had [?] $60 \%$ of samples with the given ASV/taxon, or, if the result of Fisher's exact test was significant (after multiple-testing correction by the Benjamini-Hochberg method), using the R package metagenomeSeq, and custom function super.fitZig.kv.

Results showed that the fresh cow's milk samples (both urban and rural) had significantly higher numbers of ASVs and identified taxa compared toamasi and commercially fermented milk. All three commercially fermented samples appeared similar and compared to amasi, had lower numbers of ASVs and merged taxa. The commercially fermented milk had the lowest Simpson alpha diversities. The Shannon alpha diversity was high in the three urban fresh cow's milk samples. The commercially fermented milk samples were very similar and low in diversity. The Shannon alpha diversity of the two rural fresh cow's milk samples, was markedly dissimilar (Figure 1). Principal coordinates analysis, based on Bray-Curtis distances, was used to examine the dissimilarities between different cow's milk samples' microbiota communities. The four differently sourced milk groups (rural fresh, urban fresh, amasi, and commercially fermented) were strikingly dissimilar. The home fermented milk sample amasi and the two rural fresh milk samples, were uniquely dissimilar from each other and from all the other milk samples (Figure 2).

Commercially fermented products were similarly dominated by lactic acid producing bacteria, belonging to 
the phylum Firmicutes (more than 98\% abundance) and the phylum Proteobacteria (less than $2 \%$ abundance). Theamasi sample comprised approximately $50 \%$ Firmicutes and approximately $50 \%$ Proteobacteria . Rural fresh milk 1 was comprised almost completely of Proteobacteria with small percentages of Bacteroidetes , Firmicutes and Candidatus Saccharibacteria (Figure S1).

Commercially fermented products appeared remarkably similar at genus level, with very low richness, comprising of mainly two genera:Lactococcus (more than $75 \%$ relative abundance) andLeuconostococcus (about $24 \%$ relative abundance). Theamasi sample appeared to have higher richness than commercially fermented milk, but less than the fresh milk samples. In amasi, Lactococcus had the highest relative abundance, but the genusLeuconostococcus was absent. Furthermore, in amasi, the genera Kluyvera, Citrobacter, Streptococcus ,Lactobacillus and Salmonella were present (Figure S2).

Although lactic acid producing bacteria were identified in the fermented milk products, the presence of these organism in the fresh milk samples, were inconsistent. In amasi and in the commercially fermented products, Lactococcus lactis was the most abundant organism.Lactobacillus paracasei was abundant in amasi , but almost completely absent in all the other milk samples (Figure 3).

Microbiota composition was compared in fermented (home and commercially) versus unfermented milk. Because of the relatively small number of samples used in this study, all fermented milk samples were included for statistical comparison against the unfermented milk samples.

Results for differential abundance testing clearly show significant difference in Lactococcus and Leuconostococcus species between the fermented and unfermented milk groups. There are several taxa that uniformly dominated in commercially fermented milk, contributing to its evenness and decreased diversity. Lactococcus lactis dominated in the fermented samples, including amasi .Lactococcus chungangensis had similar abundance across commercially fermented products, was absent in amasi and present at exceptionally low levels in the fresh cow's milk products.Leuconostococcus mesenteroides and Leuconostococcus pseudomesenteroides were absent in amasi . Lactobacillus paracasei was abundant in amasi, with low to no occurrence in all the other samples (Figure S3).

Potential milk pathogens were also differentially abundant between fermented and unfermented milk. Salmonella enterica in addition to being present in all fresh cow's milk samples, was also abundant inamasi , but absent in commercially fermented products.Escherichia/Shigella was present in urban fresh samples and absent in rural and fermented milk samples (Figure 3; Figure S3).

In our study, the genus Lactococcus uniformly dominated in the commercially fermented milk samples and was also abundant in theamasi sample. An important aim of our study was to compare the microbiota (at species level) of fermented versus unfermented milk products, to indicate whether fermentation (either commercially or home fermented) had significant influences on the occurrence of certain taxa.Lactococcus lactis in the fermented group, reached the highest statistical difference between the two groups. Lactococcus chungangensis, Leuconostococcus mesenteroides, Leuconostococcus pseudomesenteroides and Lactobacillus paracasei were also significantly more abundant in the fermented milk group. Potential human pathogens were identified in fresh cow's milk andamasi. Noteworthy, these organisms were absent in the commercially fermented products.

Our study was of small numbers and limited to bacteria without assessing viral, parasitic, or fungal differences. Concerns about contaminant DNA (e.g. from the laboratory environment, laboratory technicians and nucleic extraction kits) and cross contamination (e.g. DNA from other laboratory samples and sample runs) when analyzing low microbial biomass samples, have been published. ${ }^{9}$

The consumption of fermented milk appears protective in our setting. Traditional home fermentation is time consuming and supplanted by the ingestion of commercially fermented milk. Although low in diversity, commercially fermented milk appears safe, accessible and its microbiota composition appears to be consistently abundant of lactic acid producing bacteria. It is therefore not surprising that commercially soldamasi and yogurt are advised to children from one year of age in the recently updated South African Food-Based 
Dietary guidelines. ${ }^{10}$ The consumption of commercially fermented milk should be encouraged in urbanized, modern population groups, with a high prevalence of allergic disease.

\section{Acknowledgments}

The authors would like to acknowledge the ilifu cloud computing facility (www.ilifu.ac.za), used for data processing in this study. The author would further like acknowledge the input from the laboratory staff at the Centre of Proteomic and Genomic Research Laboratory, Cape Town, especially Jeanne Korsman and Enrico Roode. The author wishes to express his gratitude to all the farmers of the Mqanduli rural are in Eastern Cape and the Phillipi suburban farm, near Cape Town.

\section{Key message}

Commercially fermented milk products, although low in microbiota diversity, are safe and may be utilized as an allergy protective complementary feed in children older than 1 year of age, especially in modern, urban population groups with a high prevalence of allergic disease.

\section{References}

1. Sozańska B. Raw Cow's Milk and Its Protective Effect on Allergies and Asthma. Nutrients . 2019;11(2):469. Published 2019 Feb 22. doi:10.3390/nu11020469

2. García-Burgos M, Moreno-Fernández J, Alférez MJM, Díaz-Castro J, López-Aliaga I. New perspectives in fermented dairy products and their health relevance. J Funct Foods . 2020;72:104059. doi.org/10.1016/j.jff.2020.104059

3. Levin ME, Botha M, Basera W, et al. Environmental factors associated with allergy in urban and rural children from the South African Food Allergy (SAFFA) cohort. J Allergy Clin Immunol . 2020;145(1):415426. doi:10.1016/j.jaci.2019.07.048

4. Roduit C, Frei R, Ferstl R, et al. High levels of butyrate and propionate in early life are associated with protection against atopy. Allergy . 2019;74(4):799-809. doi:10.1111/all.13660

5. Andrews S. FastQC: a quality control tool for high throughput sequence data. https://www.bioinformatics.babraham.ac.uk/projects/fastqc. Accessed March 15, 2020.

6. Ewels P, Magnusson M, Lundin S, Käller M. MultiQC: summarize analysis results for multiple tools and samples in a single report.Bioinformatics. 2016;32(19):3047-3048. doi:10.1093/bioinformatics/btw354

7. Callahan BJ, McMurdie PJ, Rosen MJ, Han AW, Johnson AJ, Holmes SP. DADA2: High-resolution sample inference from Illumina amplicon data.Nat Methods. 2016;13(7):581-583.

8. Alishum A. DADA2-formatted 16S rRNA gene sequences for both bacteria \& archaea (Version 3). https://zenodo.org/record/3266798\#.XhxpjpMzZTY. Accessed March 15, 2020.

9. Eisenhofer R, Minich JJ, Marotz C, Cooper A, Knight R, Weyrich LS. Contamination in Low Microbial Biomass Microbiome Studies: Issues and Recommendations. Trends Microbiol . 2019;27(2):105-117. doi:10.1016/j.tim.2018.11.003

10. Du Plooy Z, Schonfeldt HC, Hall N. The role of traditional foods in food-based dietary guidelines - A South African case study on maas (cultured milk). Food Chem . 2018;238:22-28. doi:10.1016/j.foodchem.2017.04.044

Figure legends:

Figure 1 . Simpson and Shannon alpha diversity of different cow's milk samples.

Figure 2. Principle coordinates analysis (PCoA) based on Bray-Curtis dissimilarity of different cow's milk samples. 
Figure 3. Composite bar graph of lactic acid producing bacteria and pathogens with potential relevance to human health.

\section{Online supporting information :}

Figure S1. Bar plot of relative abundance of bacteria in the different cow's milk samples at phylum level, excluding low abundance taxa.

Figure S2. Bar plot of relative abundance of organisms in the different cow's milk samples at genus level, excluding low abundance taxa.

Figure S3 . Log2-transformed counts of taxa significantly different in terms of relative abundance or frequency between fermented versus unfermented samples. Taxa are merged at the lowest available taxonomic level and samples were clustered using complete linkage clustering of the Bray-Curtis dissimilarity matrix. Taxa were deemed significantly different (in terms of abundance and/or absence/presence) between fermented versus unfermented samples if they exhibited a fold change (beta coefficient) of [?] 1.5 and had an adjusted p-value of [?] 0.05 and if at least one of the two groups compared had [?] $60 \%$ of samples with the given $\mathrm{ASV} /$ taxon, or, if the result of Fisher's exact test was significant (after multiple-testing correction by the Benjamini-Hochberg method), using the $\mathrm{R}$ package metagenomeSeq.
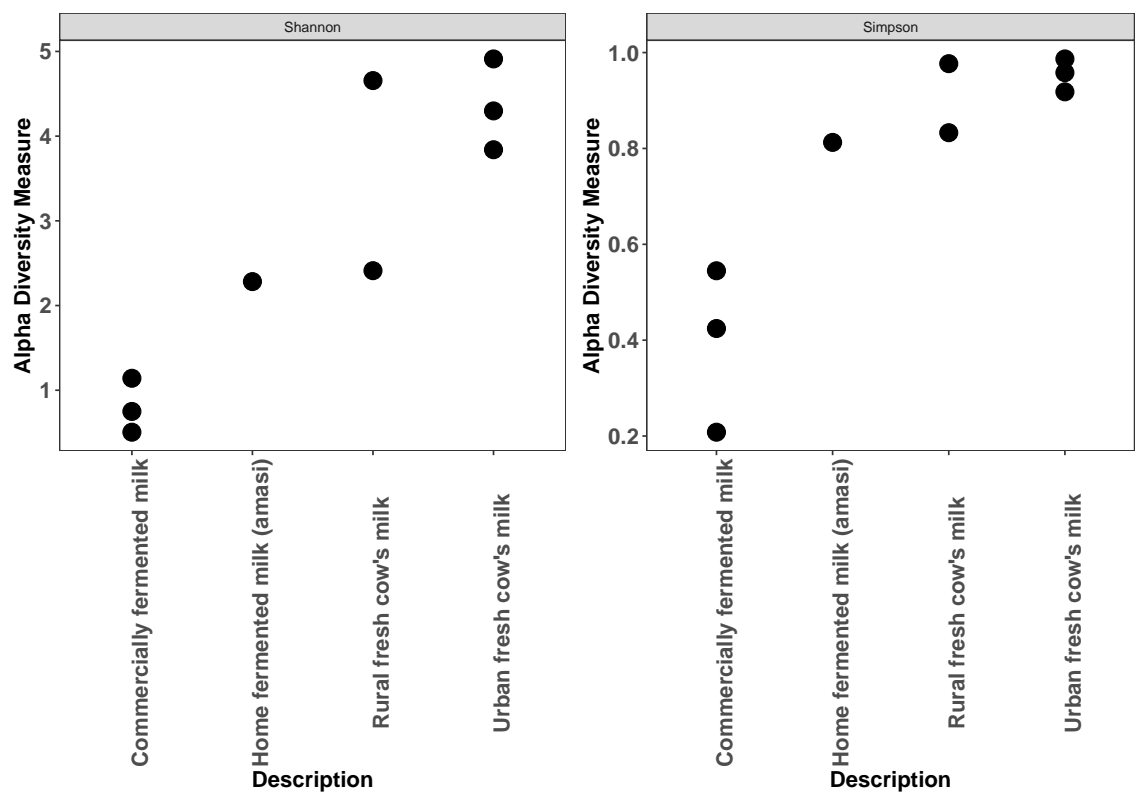

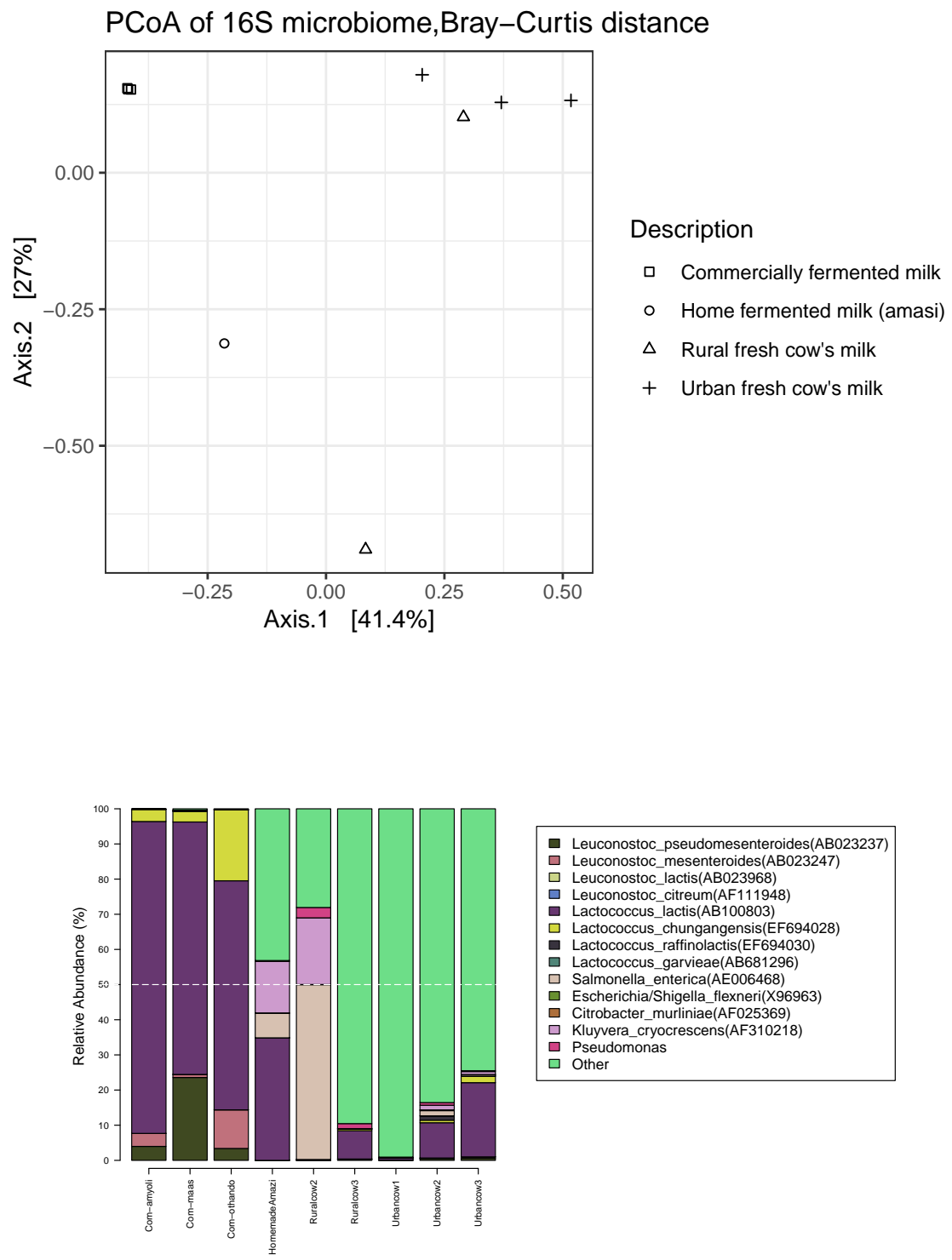

- Leuconostoc_pseudomesenteroides(AB023237)

- Leuconostoc_mesenteroides(AB023247)

Leuconostoc_lactis(AB023968)

Leuconostoc_citreum(AF111948)

Lactococcus_chungangensis(EF694028)

Lactococcus_raffinolactis(EF694030)

Lactococcus_garvieae(AB681296)

Salmonella_enterica(AE006468)

Escherichia/Shigella_flexneri(X96963)

Citrobacter murliniae(AF025369)

Kluyvera_cryocrescens(AF310218)

$\square$ Other 

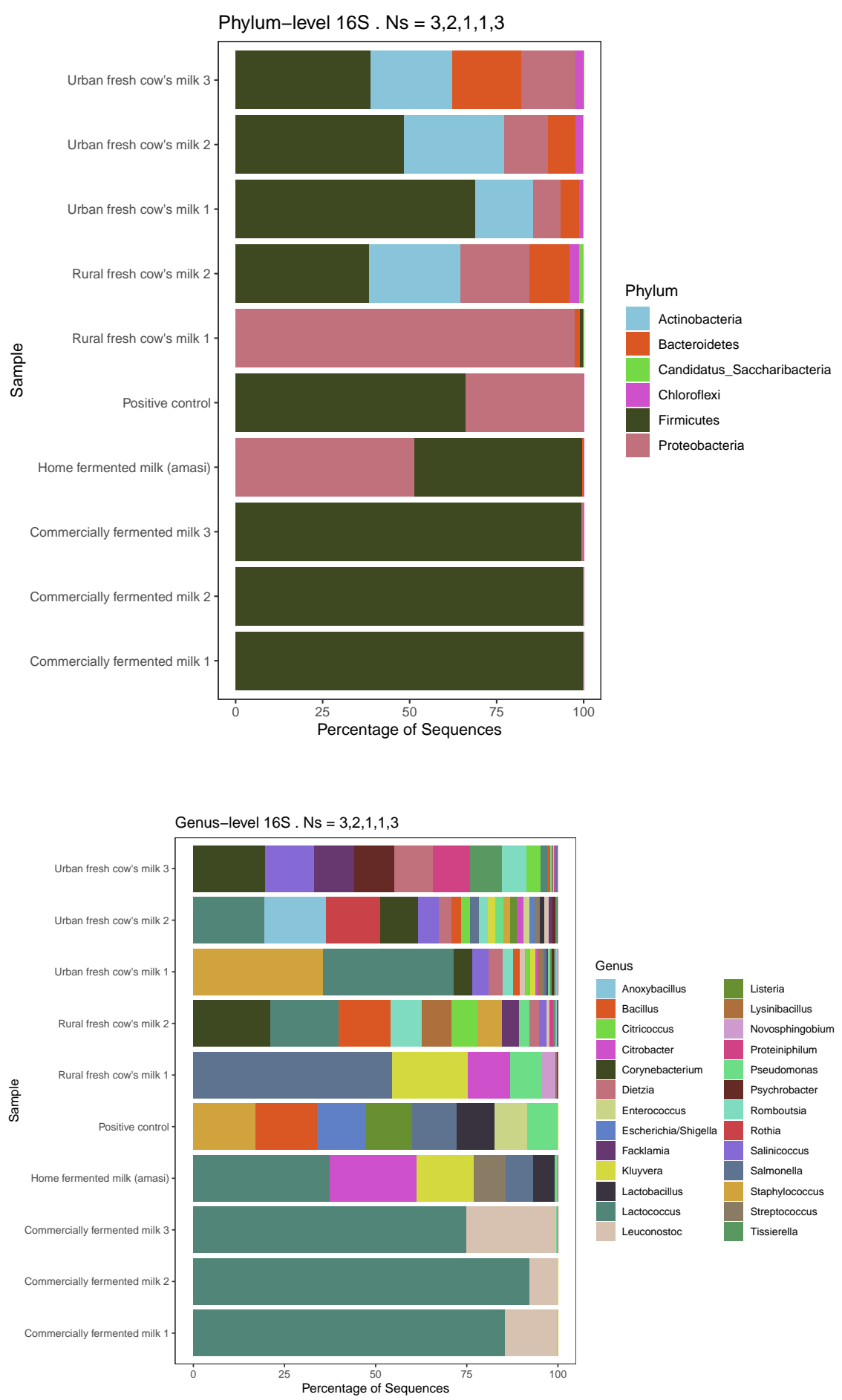


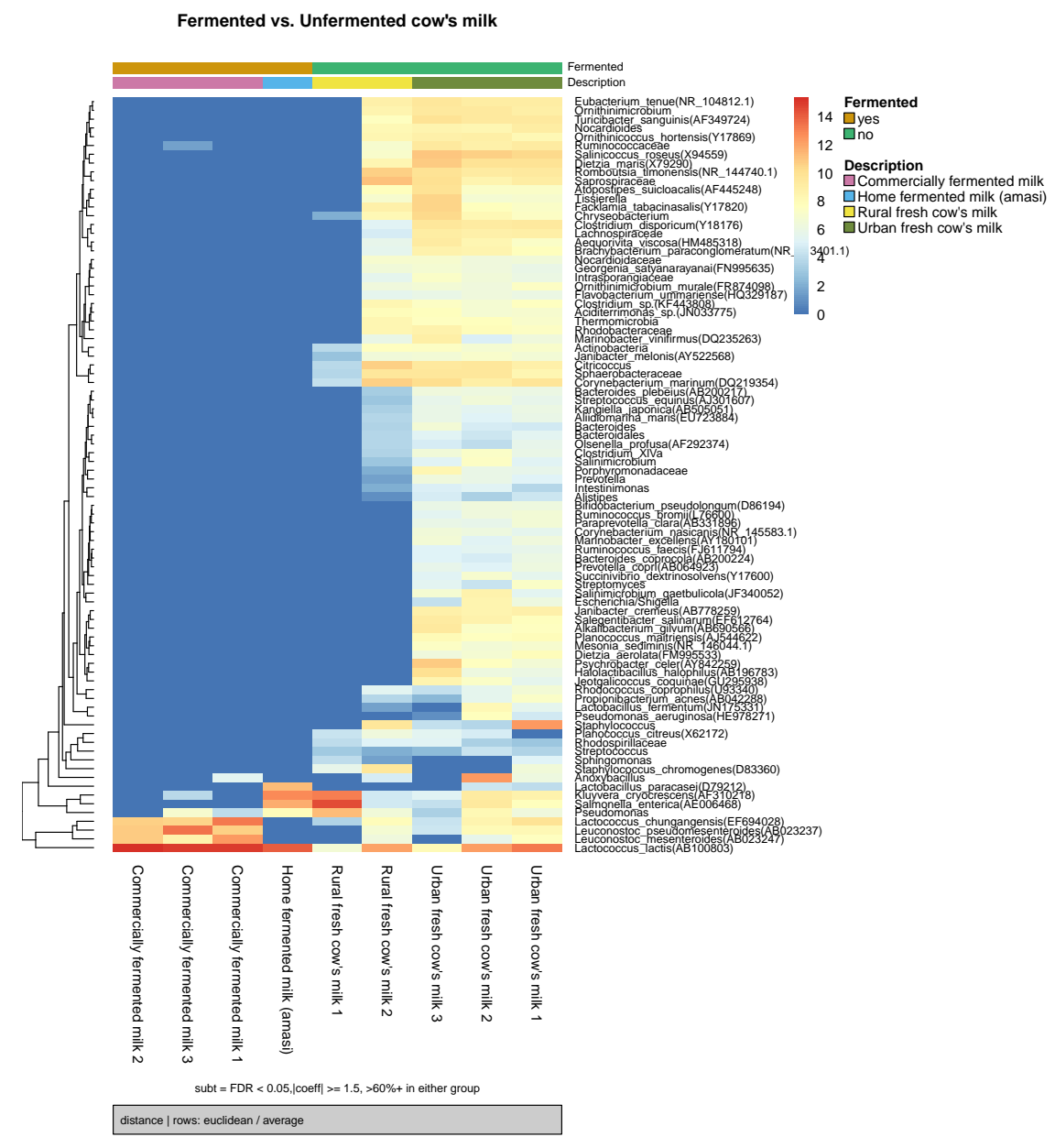

